\title{
Noise-Induced Hearing Loss: Prevention is Possible
}

\author{
Steen Gimsing, ${ }^{1, *}$ and Marianne G. Nielsen ${ }^{2}$ \\ ${ }^{1}$ Former Head of Audiology, Vejle Hospital, Vejle, Denmark \\ ${ }^{2}$ Occupational Therapist, Work Environment Consultant, ARBEjDSMILjøEksperten, Silovej 8, DK990o Frederikshavn, Denmark \\ *Corresponding author: Steen Gimsing, Former Head of Audiology, Vejle Hospital, DK7100, Vejle, Denmark. Tel: +45-22334596, E-mail: juul.gimsing@live.dk
}

Received 2015 September 3; Revised 2015 November 8; Accepted 2015 November 9.

\section{Abstract}

Background: In Denmark, the preventive measures against occupational noise-induced hearing loss (NIHL) were intensified in 1995. Apparently, no extant studies have attempted to document the effect of these measures.

Objectives: This study aims to estimate the prevalence and severity of noise-induced hearing loss after 1995 and to explain the factors that influence the official statistics regarding hearing loss.

Patients and Methods: Screening audiograms from 272 noise-exposed workers were studied in an attempt to distinguish between noiseinduced hearing loss (NIHL) and hearing loss resulting from other causes. The results were compared with deductions that could be made from official work environment statistics.

Results: Calculations based on official work environment statistics show that the proportion of NIHL among the reported cases of hearing loss has decreased, so that the proportion of non-NIHL has increased. Further, the severity of occupational NIHL has decreased. Among the 272 tested persons, 88 (32\%) presumably had NIHL. A combination of NIHL and hearing losses of other causes was found in $28(10 \%)$, while $43(16 \%)$ had a hearing loss that could not be NIHL. Normal hearing was found in 113 (42\%). Overall, roughly, one third of the tested persons had NIHL, and among the 159 cases with hearing loss, a factor other than noise played a role. The measures against NIHL have had a positive effect. When, nevertheless, the annually reported number of cases continues to rise, it is because minimal NIHL cases and cases that are not NIHL are included in the statistics. The present study suggests that the non-NIHL group may amount to about $40 \%$ of the reported cases. The official statistics should be refined to take this confounding aspect into account.

Conclusions: Despite rising figures of reported occupational hearing loss, working NBII statistics show that the NIHL problem in the Danish work environment has, in fact, decreased. In our study, we found that roughly one third (around 100 cases) of the 272 screened persons presumably had NIHL, taking into account 88 probable cases (Column B) and 44 questionable ones (Column C +16 ski slopes). About $45 \%$ of those who did not pass the screening had a hearing loss in which noise could be, at the most, a contributory factor. In 116 cases of probable or possible NIHL, the average hearing loss was only $33 \mathrm{~dB}$, and in only 11 cases (9\%) was the hearing loss considered severe enough to qualify for compensation. Our audiometric findings are, in all probability, valid for similar plants in this country and suggest that the noise problem is reasonably under control. This corroborates the deductions that can be made from the official statistics. It is evident, therefore, that NIHL incidence and severity can be reduced in countries that implement and enforce appropriate work environment legislation. However, documentation of the effect requires data storage and retrieval systems that can distinguish between NIHL and non-NIHL cases and are also able to grade the severity of NIHL.
\end{abstract}

Keywords: Noise, Workplace, Hearing Loss, Noise-Induced, Hearing, Audiometry

\section{Background}

Noise-induced hearing loss (NIHL) is a serious, widespread, but preventable occupational health hazard in many trades. In Denmark (population 5.6 million), the maximum permissible noise exposure is $85 \mathrm{dBA}$ for eight hours daily. If it is not possible to meet this requirement, ear protectors are mandatory, and if the noise level exceeds $80 \mathrm{dBA}$, exposed persons must be offered screening audiometry at "suitable intervals." When the noise exceeds $85 \mathrm{dBA}$, clinical audiometry must be offered instead. Screening audiometry is a simplified hearing test that can be carried out at the plant by trained persons. Frequently, a soundproof booth is not used, and the screening does not measure hearing better than $20 \mathrm{~dB}$ at each frequency. In addition, it cannot separate middle ear hearing loss from inner ear hearing loss. Clinical audiometry, on the other hand, is a complete audiometric work-up in a soundproof booth, but can only reliably be carried out by fully educated audiometric technicians.

The working environment authority (WEA) supervises all Danish work environments, while the national board of industrial injuries (NBII) handles compensation issues. It is compulsory for physicians to report cases of possible occupational injuries to the WEA. If a disability caused by occupational NIHL amounts to $5 \%$ or more, it qualifies for compensation. Very troublesome tinnitus, combined with NIHL, may also be compensated. A dis- 
ability set at $5 \%$ will, for instance, entitle a 60 -year-old person to the equivalent of 4.500 EUR in compensation, while a younger person will receive more.

Consultancies licensed by the WEA often assist companies in their work environment surveillance, and if the WEA orders shortcomings to be rectified, the company in question must make use of a licensed consultancy to correct the problem. Such consultancies employ a wide range of technicians and health professionals.

The prevention of NIHL has received much attention for at least 30 years, and in 1995 the WEA launched a 10-yearlong campaign to intensify the effort against NIHL. The WEA statistics have shown a decline in the number of annually reported NIHL cases from about 2400 in 1995 to about 1700 in 2005; however, since then, the number has been rising steadily, reaching 2675 cases in 2013 . Nevertheless, these rising figures do not reflect reality and, to professionals with long experience in public hearing health care, it is obvious that the true development has moved in the opposite direction: There are fewer new NIHL cases, and the hearing losses are much smaller than before. Evidence of the latter can be seen in the latest NBII annual report (1), which shows that the average amount paid in compensation for NIHL in the 2011 - 2013 interval was 15\% lower than in the 2007-2010 period. This favorable development is also evident in the general population, where the previous cavalier attitude toward harmful noise levels has given way to the conscientious use of ear protectors, often even during leisure activities.

The fact that NIHL Figures, nevertheless, are rising can be attributed to two causes: in the first place, preventive work itself leads to the detection of very small NIHLs, ones that would otherwise remain unreported, and to the detection of hearing losses that are not NIHL so that they, erroneously, enter the statistics. In the second place, the prospect of compensation invites unfounded cases to be reported.

Accordingly, the proportion of acknowledged NIHL among the reported cases should decline, and so should the average amount of disability apportioned to acknowledged NIHL cases. Indeed, there is evidence of this in the WEA data: although the criteria for acknowledging NIHL were eased in 2005, precluding the comparison of data before and after that date, it is evident (1) that the acknowledgement rate dropped from $55 \%$ in the 2007 - 2009 interval (2,965 of 5,417 cases) to $41 \%$ (3,024 of 7,298 cases) in the $2011-2013$ period $(\mathrm{P}<0.01)$, proving that fewer of the reported cases were actually NIHL. In addition (personal communication), in the $2007-2009$ interval, $54 \%$ of the acknowledged NIHL cases $(1,598$ of 2,987 ) received compensation, while it was $48 \%$ in the 2011 - 2013 interval (1,458 of 3,064), clearly indicating that the NIHL cases in the latter period were milder ( $P$ $<0.01$ ). These two sets of data indicate a favorable development with regard to the noise problem in Danish work environments, but, to our knowledge, this has not been pointed out before. Since the NIHL problem is waning, it is to be expected that hearing losses found in noise-exposed groups in this country, to a considerable extent, will have causes other than noise.

\section{Objectives}

In 2013, we had the opportunity to look into this situation, as the second author, who is an occupational therapist in a licensed work environment consultancy, carried out screening audiometry at a major industrial plant in accord with the legislation outlined above. The noise there primarily stemmed from large fiberglass objects being polished with angle grinders, generating noise levels reaching $100 \mathrm{dBA}$. An analysis of the audiograms permitted us to assess to what extent noise or other factors can explain hearing losses found in these employees.

\section{Patients and Methods}

Two hundred and sixty-three males between 21 and 65 years (mean 40.5) and nine women aged between 29 and 53 (mean 43.8) were tested in a portable soundproof booth placed in a suitable room at the plant. One hundred and twenty-five persons (46\%) could report the likely duration of their noise exposure, the mean of which was 15.3 years, and ranging from less than one year to 45 years. It was impossible, in the individual cases, to assess the intensity of the noise due to widely different exposure patterns among individuals and over time in the individual cases. The air conduction thresholds of each ear were determined, down to $20 \mathrm{~dB}$ hearing level at 11 frequencies, from 125 to 8,000 Hz. The median values in Database A of ISO 1999 (2) were used to define normal hearing. Since we did not test below $20 \mathrm{~dB}$, hearing was considered normal in males aged 55 or more if they could hear at the frequencies up to $3,000 \mathrm{~Hz}$ at $20 \mathrm{~dB}$ and at $4,000 \mathrm{~Hz}$ and 6,000 $\mathrm{Hz}$ at 25 and $30 \mathrm{~dB}$, respectively. In females and males younger than 55, normal hearing was defined as hearing not poorer than $20 \mathrm{~dB}$ at all frequencies below $8000 \mathrm{~Hz}$. The data processing was based on the frequencies of 250 ; $500 ; 1,000 ; 3,000 ; 4,000$ and $6,000 \mathrm{~Hz}$. The following criteria were used to define deviation from normal hearing: A threshold at $25 \mathrm{~dB}$ or poorer at two or more frequencies in the mid/low frequency range (250 to $2,000 \mathrm{~Hz}$ ), or a threshold elevation of at least $5 \mathrm{~dB}$ at one frequency between 3,000 and 6,000 Hz. Asymmetrical hearing was defined as a $15 \mathrm{~dB}$ difference between the right and left ear at two or more frequencies from 2,000 to $6,000 \mathrm{~Hz}$. Symmetrical cases had almost identical hearing in the two ears, so that the calculations in both the symmetrical and asymmetrical group were based on the better ear. Since another study (unpublished data) showed that the average of the thresholds at 1,000;2,000 and 3,000 has a higher correlation with hearing disability in NIHL than any other audiogram parameter; we used this figure to analyze the correlation between exposure and hearing loss. Those workers who did not pass the hearing screening as described above were advised to consult an otolaryngologist for further evaluation. 


\section{Results}

The audiogram was symmetrical in 246 (90\%) cases and asymmetrical in 26 (10\%). Table 1 summarizes the audiometric results. Column A shows that 113 persons (42\%) had normal hearing in both ears. Columns B and $C$ show that 88 persons (32\%) had an audiogram consistent with NIHL, while in 28 cases (10\%) the hearing loss could only partially be NIHL. In 43 cases (16\%) listed in Column D, NIHL was unlikely. In the 116 cases with NIHL or partial NIHL (Columns B and C), the mean hearing loss at the "noise frequencies" $(4,000$ or $6,000 \mathrm{~Hz})$ was only $33 \mathrm{~dB}$. The 125 persons in whom the duration of the noise exposure was known had audiograms identical to those with unknown exposure times. When individuals with pure high frequency losses $(n=119)$ were compared with the remaining 153, there was no difference with regard to reported exposure time, and in neither category was there a correlation between the duration of exposure and hearing loss quantified as the 1,000; 2,000 and 3,000 $\mathrm{Hz}$ average. In contrast, there was a significant age difference $(\mathrm{P}<0.01)$, as the average age in the high frequency group was 43.1 years, while it was 38.7 in the other group.

Table 1. Characteristics of the Audiograms in 272 Individuals Screened for Noise-Induced Hearing Loss

\begin{tabular}{lccccc}
\hline & $\begin{array}{c}\text { Normal } \\
\text { Hearing }\end{array}$ & NIHL & $\begin{array}{c}\text { NIHL+ } \\
\text { Other Cause }\end{array}$ & $\begin{array}{c}\text { Not } \\
\text { NIHL }\end{array}$ & Total \\
\hline $\begin{array}{l}\text { Audiogram } \\
\text { configuration }\end{array}$ & A & B & C & D & E \\
Normal & $113(42)$ & NA & NA & NA & 113 \\
4-6 kHz dip & NA & $88(32)$ & NA & NA & 88 \\
\hline 4-6 kHz dip + asymmetry & NA & NA & $7(2)$ & NA & 7 \\
3 kHz dip only & NA & NA & NA & $6(2)$ & 6 \\
$\begin{array}{l}\text { 4-6 kHz+low/mid frq. } \\
\text { loss }\end{array}$ & NA & NA & $21(8)$ & NA & 21 \\
Ski slope & NA & NA & NA & $16(6)$ & 16 \\
Low/mid frq. loss only & NA & NA & NA & $21(8)$ & 21 \\
Total & $113(42)$ & $88(32)$ & $28(10)$ & $43(16)$ & 272 \\
\hline
\end{tabular}

Abbreviations: Asymmetry, side difference between the two ears in the high frequency range; Low/mid frq., hearing loss at the low and middle frequencies; kHz, Kilohertz; NA, not available; NIHL, noise-induced hearing loss.

\section{Discussion}

The typical NIHL audiogram shows a distinct high tone hearing loss centered at 4,000 or 6,000 Hz (Figure 1), often referred to as a "noise notch," while hearing at 1,000 and $2,000 \mathrm{~Hz}$ is largely unaffected. Hearing at 3,000 $\mathrm{Hz}$ should be close to the average of 2,000 and $4,000 \mathrm{~Hz}(3,4)$. NIHL will always display this pattern, but other types of hearing loss, especially hereditary types, can have the same shape. In NIHL, hearing in the two ears will be symmetrical (5), unless a specific event, such as an explosion near one ear,
Figure 1. The Expected Audiogram of a 55-Year-Old Man After 30 Years in Noise at $95 \mathrm{dBA}$

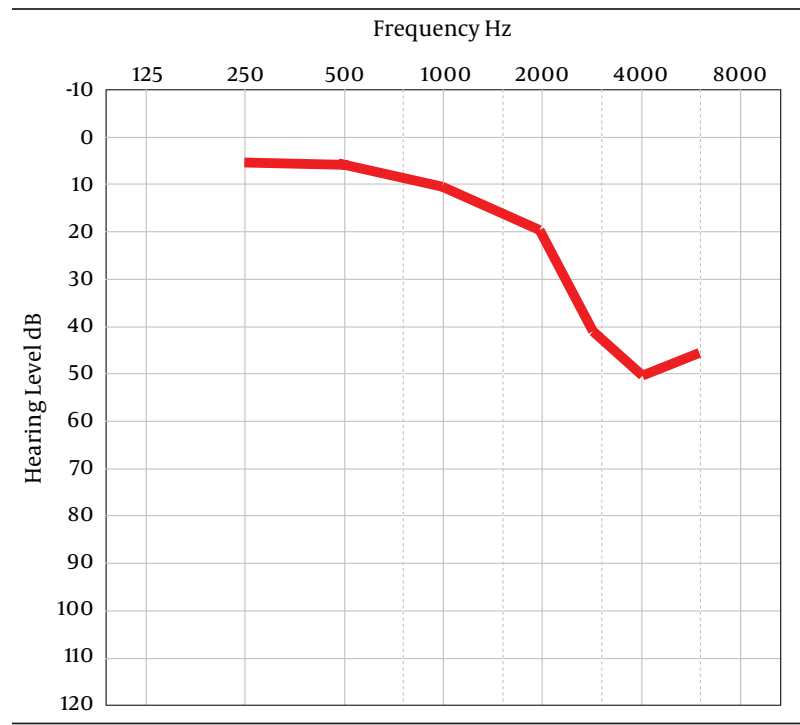

Predicted from ISO-1999.

can account for a side difference. The development of NIHL follows a characteristic course, shown graphically by Taylor et al. (6) and in tabular form by ISO-1999 (2): during the first ten years of exposure, there is a rapid deterioration at $4,000 \mathrm{~Hz}$ and a parallel, but milder one, at 3,000 Hz. The deterioration at both frequencies saturates after ten years so; after that time, little progression occurs despite continued exposure. In contrast, at 2,000, and to some extent at 1,000 $\mathrm{Hz}$, a linear loss develops gradually and slowly. Accordingly, a 4,000 or $6,000 \mathrm{~Hz}$ loss that progresses beyond ten years and often reaching 80 - $90 \mathrm{~dB}$ should be suspected to have causes other than noise.

Therefore, an asymmetry between the two ears, a hearing loss at a single frequency below or above 4,000 $6,000 \mathrm{~Hz}$, a marked deterioration starting at 1,000 or $2,000 \mathrm{~Hz}$, or a low and/or middle frequency loss indicates that factors other than noise have influenced the audiogram. However, such factors often act in combination with noise, so that the resulting audiogram becomes a combination of NIHL and other causes.

When there is asymmetry, a tumor of the acoustic nerve (acoustic neuroma) must always be ruled out. For this reason, our criterion for asymmetry was adopted from an acoustic neuroma protocol (7). A hearing loss in the low/ mid frequency range $(250-2,000 \mathrm{~Hz})$ cannot be NIHL and rather represents middle ear disease, otosclerosis, Meniere's disease, or hereditary conditions. High tone hearing losses with configurations that are not consistent with NIHL are usually of a hereditary nature. The so-called ski-slope audiograms (Figure 2), which are often confused with NIHL, represent several types of progressive hereditary hearing loss characterized by a high frequency loss that is conspicuously wider and more rounded than a "noise notch" (3). At an early stage it may be fully identical to NIHL, but as it progresses, 
deterioration at 2,000 and 3,000 $\mathrm{Hz}$ will bring about the typical appearance. Finally, it must be kept in mind that the normal age-dependent deterioration of hearing also manifests itself in the high frequencies. Tables of normal hearing at different ages can be found in ISO-1999 (2).

The findings of this study are, in all probability, fairly representative of present-day conditions in Danish industry, and therefore it is of interest to consider how our data might figure in the WEA statistics.

It is often the case that a person's hearing loss is reported to the WEA as suspected NIHL simply if the person has been noise exposed. Therefore, the 159 persons listed in Columns B, C and D who did not have normal hearing could easily have entered the statistics. In that case, the WEA could reject the 43 cases in Column D because the configuration of the audiograms would argue against NIHL, but it is, nevertheless, conceivable that some of the 16 ski slopes would be acknowledged. A substantial number, but not all, of the 88 in Column B would be accepted, but in the individual case it would depend on a detailed examination of all relevant information and documentation. Acknowledgement requires a noise exposure of at least five years in $85 \mathrm{dBA}$, an audiogram consistent with the exposure, and no evidence of a more likely cause of the hearing loss. Several of the 28 persons in Column $\mathrm{C}$ definitely would not satisfy these criteria, but it is impossible to say how many. Accordingly, it is evident that far from all 116 cases of probable or possible NIHL (Columns $\mathrm{B}$ and $\mathrm{C}$ ) of the 159 reported (73\%) would be acknowledged, but, at any rate, the figure would be higher than 41 $\%$ of the NBII statistics. The two figures do not necessarily disagree, since the persons in our study were drawn from a very noisy industry that would necessitate expecting a high NIHL prevalence.

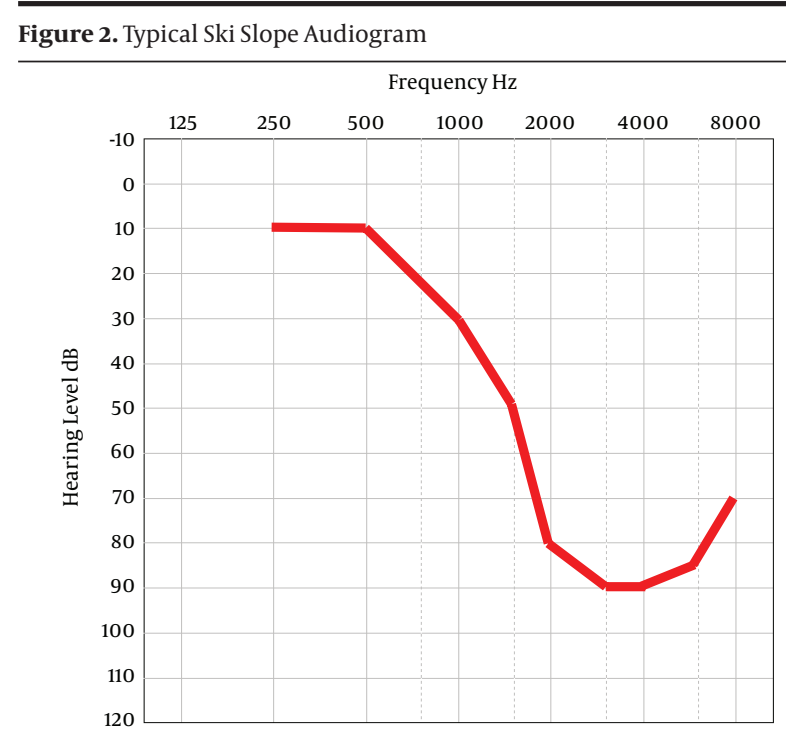

Actual audiogram of 65-year-old man with only minimal noise exposure Note the rounded shape of the hearing loss between 2,000 and 8,000 Hz and the pronounced loss at both 2,000 and 3,000 Hz.
Acknowledgement of NIHL only qualifies for compensation if the disability ascribed to NIHL, as mentioned, amounts to at least 5\%. In Denmark, NIHL disability is set by the procedure devised by Salomon et al. (8), which defines $5 \%$ auditory disability as the condition where a person cannot manage a two-person conversation in background noise without lip reading. In the individual case, this is determined by otolaryngologists who specialize in audiology using word recognition measurements in quiet and in background noise. We have analyzed 350 such affidavits, and on this background we estimate that only 11 of the 116 persons ( $9 \%$ ) in Columns B and C would attain $5 \%$ disability. In addition, several of them would not have occupational NIHL, but rather hearing loss caused by leisure noise or hereditary conditions.

Seventy-one (Columns C and D) of the 159 who failed the screening (45\%) had a hearing loss that was definitely influenced or caused by factors other than noise. This agrees reasonably with the $53 \%$ reported in a comparable study (9), indicating that about $50 \%$ of those who fail an industrial hearing screening do not have NIHL. When the purpose is to detect NIHL, such a high rate of incidental findings is undesirable, but since they sometimes reflect serious conditions that require intervention, detecting them may be at least as important as detecting NIHL, as far as the individual is concerned. Nevertheless, the high rate of incidental findings has two important general implications for industrial hearing screening: it erodes the efficiency of the screenings designed to monitor NIHL occurrences, and it makes it imperative that those who fail be seen subsequently by an otolaryngologist.

Since the relationship between noise exposure (intensity and duration) and NIHL is a well-established fact, it is interesting that our study failed to demonstrate such a correlation. There may be several explanations for this: one is that many of the hearing losses in our study had causes other than noise, at least exclusively. Another is that exposure time itself does not imply that the noise level has been harmful, so many persons, despite long exposure, may not have been at risk of NIHL. Since in about $50 \%$ of the cases the exposure had begun in the late "nineties," when the preventive measures were becoming effective, this group may be large enough to mask the effect of exposure time in individuals who had been exposed to harmful noise levels. This may also explain the age differences between individuals with and without a hearing loss entirely in the high frequencies: these persons were old enough to have acquired their NIHL at a time when the noise problem in the work environment was severe.

\section{Acknowledgments}

The first author is a part-time audiology consultant to the National Board of Social Appeals. The second author is joint owner of the company that carried out the hearing screening. 


\section{Footnote}

Authors' Contribution:Data analysis and preparation of manuscript: Steen Gimsing; all hearing tests and manuscript co-editing: Marianne G. Nielsen.

\section{References}

1. National Board of Industrial Injuries. 2013; Available from: www. ask.dk/da/Statistik/Arbejdsskader/Arbejdsskadestatistik.aspx.

2. The International Organization for Standardization . AcousticsDetermination of occupational noise exposure and estimation of noise-induced hearing impairment. 3 ed. Geneva, Switzerland: The International Organization for Standardization (ISO 1999); 1990. p. 23.

3. Gimsing S. [Diagnostics on noise-induced hearing loss]. Ugeskr Laeger. 2014;176(31):1445-8. [PubMed: 25292321]

4. Gurgel RK, Popelka GR, Oghalai JS, Blevins NH, Chang KW, Jackler
RK. Is it valid to calculate the 3-kilohertz threshold by averaging 2 and 4 kilohertz? Otolaryngol Head Neck Surg. 2012;147(1):102-4. doi:10.1177/0194599812437156. [PubMed: 22301102]

5. Dobie RA. Does occupational noise cause asymmetric hearing loss? Ear Hear. 2014;35(5):577-9. doi: 10.1097| AUD.0000000000000043. [PubMed:24879031]

6. Taylor W, Pearson J, Mair A, Burns W. Study of Noise and Hearing in Jute Weaving. J Acoust Soc Am. 1965;38:113-20. [PubMed: 14347600]

7. Gimsing S. Vestibular schwannoma: when to look for it? J Laryngol Otol. 2010;124(3):258-64. doi: 10.1017/S0022215109991423. [PubMed:19922702]

8. Salomon G, Parving A, Danielsen K. [Definitions and graduation of hearing disorders and handicapped communication for use in medico-legal compensation]. Ugeskr Laeger.1985;147(8):685-91. [PubMed: 3157253]

9. Dobie RA, Archer RJ. Results of otologic referrals in an industrial hearing conservation program. Otolaryngol Head Neck Surg. 1981;89(2):294-301. [PubMed: 6787529] 